\title{
Graph Related to Cubed Commutativity Degree
}

\author{
N. M. Mohd Ali ${ }^{*}{ }^{*}$, M. Abdul Hamid ${ }^{2}$, N. H. Sarmin ${ }^{1}$ and A. Erfanian 3 \\ ${ }^{1}$ Department of Mathematical Sciences, Faculty of Science, Universiti Teknologi Malaysia, 81310 UTM \\ Johor Bahru, Johor, Malaysia \\ ${ }^{2}$ Faculty of Computer and Mathematical Sciences, Universiti Teknologi MARA, 40450 Shah Alam, Selangor \\ ${ }^{3}$ Department of Pure Mathematics, Faculty of Mathematical Sciences, Ferdowsi University of Mashhad, \\ Mashhad, Iran
}

Let $G$ be a finite group and $T^{3}(G)$ be the set of third power of commuting element in $G$ i.e $T^{3}(G)=\left\{x \in G \mid(x g)^{3}=(g x)^{3}\right\}$. We define a graph, $\Gamma$ with the vertex set $G \backslash T^{3}(G)$ in which two vertices $x$ and $y$ are joined by an edge (connected) if $(x y)^{3} \neq(y x)^{3}$. This graph is called as graph related to cubed commutativity degree. In this research, we study this graph and find a characterization of finite groups in term of this graph.

Keywords: graph, cubed commutativity degree

\section{INTRODUCTION}

Recently there are tremendous research to construct a graph by a group or semigroup. Since algebraic graph theory has close links with group theory, algebraic methods are applied to problem about graphs or vice versa. A new graph called graph related to conjugacy class was introduced by (Bertram et al., 1990) by considering its vertices are non-central conjugacy classes, where two vertices are adjacent if the cardinalities are not coprime. As a consequence, numerous works have been done on this graph and many results have been achieved. See (Bianchi et al., 1992; Chillag et al., 1993; You et al., (2005); Moreto, et al., 2005) for more details.

In 2012, (Erfanian \& Tolue, 2012) introduced the relative non nil-n graph, $\Gamma_{H, G}^{(n)}$ of a finite group $G$. It is a graph with vertex set $G \backslash C_{G}^{(n)}(H)$ and two distinct vertices $x$ and $y$ are adjacent if at least one of them belongs to $H$ and $[x, y] \notin Z_{n-1}(G)$, where the subgroup $C_{G}^{(n)}(H)$ contains $g \in G$ such that $[g, h] \in Z_{n-1}(G)$ for all $h \in H$.
They proved that two $n$-isoclinic groups which are not nil- $n$ groups have isomorphic graphs under special conditions. In the same year, (Erfanian \& Tolue, 2012) introduced a new graph which is called a conjugate graph. The vertices of this graph are non-central elements of a finite non-abelian group. Two vertices of this graph are adjacent if they are conjugate. Moreover, (Bianchi et al. 2012) studied the regularity of the graph related to conjugacy class and provided some results.

Furthermore, the orbit graph is a result of generalization of conjugate graph. This graph was firstly introduced by (Omer et al., 2014). The number of vertices of an orbit graph is $\left|V\left(\Gamma_{G}^{\Omega}\right)\right|=|\Omega|-|A|$ where $\Omega$ is a disjoint union of distinct orbits under the action of $G$ on the set $\Omega$, while $A=\{v \in \Omega \mid v g=g v, g \in G\}$. Two vertices of this graph are linked by an edge if and only if there exists $g \in G$ such that $g w_{1}=w_{2}$, where $w_{1}, w_{2} \in \Omega$ . A year later, El-sanfaz and Sarmin found the generalized conjugacy classes graph of metacyclic 2-groups of positive type of nilpotency class at least three. 


\section{METHODS}

This section focuses on new graph which is related to the cubed commutativity degree, denoted by $\Gamma_{3}(G)$. Let $w(x, y)$ be a word and $w$ be the variety of groups defined by the law $w(x, y)=1$. Suppose that $G$ is a group which is not in $w$. Let $T(G)=\{x \in G \mid w(x, g)=w(g, x)=1$, for all $g \in G\}$. A graph, $\Gamma$ is defined as follows;

Definition 1. Thevertex set $V=V(\Gamma)$ of $\Gamma$ is the set $G \backslash T(G)$ and two vertices $x, y \in V$ are joined by an edge if $w(x, y) \neq 1$.

Note that to avoid isolated vertices, the vertex set is taken as the elements of $G$ outside $T(G)$. If $w(x, y)=[x, y]=x^{-1} y^{-1} x y$, the graph $\Gamma(G)$ is the noncommuting graph investigated in (Abdollahi et al., 2006). Let $n$ be a positive integer. One can investigate the graph of a group using the word $w(x, y)=\left[x, y^{n}\right]$. The variety of groups defined by the word $\left[x, y^{n}\right]$ coincides with the variety of groups defined by the word $(x y)^{n}(y x)^{-n}$. So, one can also investigate the graph of a group using the word

Example 1. Let $G=D_{5}$, since $T^{3}(G)=\bigcap_{x \in G} T_{G}^{3}(x)$, then $T^{3}\left(D_{5}\right)=e . \quad$ Therefore, $\quad V\left(\Gamma_{3}\left(D_{5}\right)\right)=G \backslash\{e\}$ i.e $V\left(\Gamma_{3}\left(D_{5}\right)\right)=\left\{a, a^{2}, a^{3}, a^{4}, b, a b, a^{2} b, a^{3} b, a^{4} b\right\} . \quad B y$ using definition of graph related to the cubed commutativity degree, two elements are joined by an edge if $(x y)^{3} \neq(y x)^{3}$, thus the graph of $\Gamma_{3}\left(D_{5}\right)$ is produced and presented in the following. $w(x, y)=(x y)^{n}(y x)^{-n}$. Therefore, a special word has been considered in this section.

Now, suppose that $G$ is a group such that $T^{n}(G)$ is a proper subgroup. The vertex set $V=V(\Gamma)$ of the graph $\Gamma:=\Gamma(G)$ is the set $G \backslash T^{n}(G)$ and two elements $x, y \in V$ are joined by an edge if $(x y)^{n} \neq(y x)^{n}$.

The following is the definition of graph related to the cubed commutativity degree.

Definition 2.Let $G$ be a finite group and $T^{3}(G)$ be the set of third power of commuting elements in $G$, i.e $T^{3}(G)=\left\{x \in G \mid(x g)^{3}=(g x)^{3}\right\}$. Then the graph related to the cubed commutativity degree, $\Gamma_{3}(G)$ is defined as a graph whose vertices are non central elements in $G$ but not in $T^{3}(G)$, that is $V\left(\Gamma_{3}(G)\right)=G \backslash T^{3}(G)$. Note that $V:=V\left(\Gamma_{3}(G)\right)=\left\{g \in G \mid(x g)^{3} \neq(g x)^{3}\right.$ for some $\left.x \in G\right\}$ and two vertices are connected if $(x y)^{3} \neq(y x)^{3}$.

The following example is given to illustrate the definition of graph related to cubed commutativity degree.

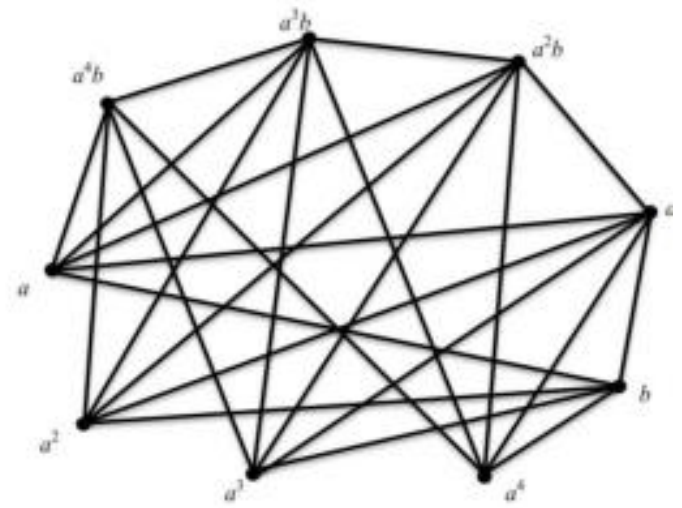

FIGURE 1: $\Gamma_{3}\left(D_{5}\right)$

\section{RESULTS AND DISCUSSIONS}

Throughout this research, $G$ is assumed as finite group, $n=3$ and $\Gamma:=\Gamma_{3}(G)$. Some results have been 
investigated on this graph starting with the following lemma.

Lemma 1. If $G$ is not abelian, then $\Gamma$ has no isolated vertex.

Proof. Assume that $x \in V$ is an arbitrary vertex. Since $x \in G \backslash T^{3}(G)$, so $x \notin T^{3}(G)$ and therefore there exists an element $g \in G$ such that $(x g)^{3} \neq(g x)^{3}$. Note that $g$ is also a vertex since $g \in G$ and $g \notin T^{3}(G)$. Therefore, there is an edge between $x$ and $g$. So, $x$ is not an isolated vertex.

Remarks If $G$ is abelian then $T^{3}(G)=G$. Next, groups with complete graphs are classified.

Theorem 1. The graph $\Gamma$ is a complete graph if and only if $H:=C\left(G^{3}\right)$ is abelian of odd order with index 2, $x^{2}=1, h^{x}=h^{-1}$, for all $x \in G \backslash H$ and all $h \in H$.

Proof. Let $x$ be any element of $G$ outside $H$. Then $G=H \bigcup x H$ and $V(\Gamma)=G \backslash H=x H$. Let $x h_{1}$ and $x h_{2}$ be two distinct vertices of $\Gamma$, where $h_{1}, h_{2} \in H$. Then $\left(x h_{1} x h_{2}\right)^{3} \neq\left(x h_{2} x h_{1}\right)$. Otherwise, $\left(h_{1}^{x} h_{2}\right)^{3}=\left(h_{2}^{x} h_{1}\right)^{3}$. So $\left(h_{1}^{-1} h_{2}\right)^{3}=\left(h_{2}^{-1} h_{1}\right)^{3} \quad$ which implies that $\left(h_{1}^{-1} h_{2}\right)^{6}=1$, contradicting the hypothesis on the order of $H$. Therefore, $x h_{1}$ and $x h_{2}$ are adjacent and $\Gamma$ is a complete graph.

Suppose that $\Gamma$ is a complete graph. Suppose that $x \neq x^{-1} \quad$ for $\quad$ some $\quad x \in G \backslash H$. Then since $\left(x^{-1} x\right)^{3}=\left(x x^{-1}\right)^{3}, x$ is not adjacent to $x^{-1}$, contradicting $\operatorname{diam}(\Gamma)=1$. Hence, $x^{2}=1$ for all $x \in G \backslash H$. For all $h \in H$ and for all $x h \in G \backslash H$, we have $x h \in G \backslash H$ and so $(x h)^{2}=1$. Therefore, $h^{x}=h^{-1}$. To see that $H$ is abelian, let $\quad h_{1}, h_{2} \in H . \quad$ Then $\left(h_{2} h_{1}\right)^{-1}=h_{1}^{-1} h_{2}^{-1}=h_{1}^{x} h_{2}^{x}=\left(h_{1} h_{1}\right)^{x}=\left(h_{1} h_{2}\right)^{-1}, \quad$ for $\quad$ all $x \in G \backslash H$, and so $h_{1} h_{2}=h_{2} h_{1}$. Hence, $H$ is abelian. Now, we prove that $|G / H|=2$. Since $G$ is not an elementary abelian 2-group i.e not all element have order two, there exists $h \in H$ such that $h^{2} \neq 1$. If $|G / H|>2$, then there exist $x_{1} x_{2} \in G \backslash H$ such that $x_{1} x_{2}=x_{1}^{-1} x_{2} \notin H$

$$
\text { Hence, } \quad h^{x_{1} x_{2}}=h^{-1} \quad \text { and } \quad \text { so }
$$
$h^{-1}=h^{x_{1} x_{2}}=\left(h^{x_{1}}\right)^{x_{2}}=\left(h^{-1}\right)^{x_{2}}=h$, which is contradiction. Hence, $|G / H|=2$. Finally, we show that $|H|$ is odd. Suppose there exists a non-identity $h \in H$ such that $h^{3}=1$ and let $x \in G \backslash H$. Then $(x x h)^{3}=\left(x^{2} h\right)^{3}=h^{3}=1$ and $(x h x)^{3}=\left(x^{2} h^{x}\right)^{3}=\left(h^{-1}\right)^{3}=1$. Hence, $x$ and $x h$ are not connected, contradicting to $\operatorname{diam}(\Gamma)=1$. Therefore, | $H \mid$ is odd. The proof then follows.

Now, we prove some results when $\left[G: C\left(G^{3}\right)\right]=2$.

Lemma 2. Suppose that $H:=C\left(G^{3}\right)$ has index 2 and let $x \in G \backslash H$ and $h \in H$ such that $h^{3} \in C(x)$. Then $h^{3} \in Z(G)$. Therefore, $A:=\left\{h \in H \mid h^{3} \notin Z(G)\right\}$ is nonempty, and for all $h \in H, x$ and $h x$ (as well as $x$ and $x h$ ) are adjacent if and only if $h \in A$. Also, the graph $\Gamma$ is $|A|$ - regular.

Proof. Let $g \in G$. It is clear that if $g \in H$, then $g h^{3}=h^{3} g$. Suppose that $g=x h_{1}$ for some $h_{1} \in H$. Thus, $g h^{3}=\left(x h_{1}\right) h^{3}=x\left(h_{1} h^{3}\right)=x\left(h^{3} h_{1}\right)=h^{3}\left(x h_{1}\right)=h^{3} g$, and therefore $h^{3} \in Z(G)$, which proves the first assertion. Now note that $(x h x)^{3}=(h x x)^{3}$ if and only if $x h x^{2} h x^{2} h x=h x^{2} h x^{2} h x^{2}$ if and only if $x^{5} h^{3} x=x^{4} h^{3} x^{2}$ if and only if $x h^{3}=h^{3} x$ if and only if $h^{3} \in C(x)$. Thus, $x$ and $h x$ are adjacent if and only if $h \in A$. To see that $A$ is non-empty, note that since $x \in H$ and $H=C\left(G^{3}\right)=T^{3}(G)$, there exists $g \in G$ such that $(x g)^{3} \neq(g x)^{3}$. Hence, $\Gamma$ has at least one edge. Suppose 
that $(x, h x)$, for some $h \in H$ is an edge. Then by the above observation, $h^{3} \notin Z(G)$ and so $A \neq \phi$. Since $x$ and $h x$ are adjacent if and only if $h \in A$, thus all vertices of the graph have degree of $|A|$. Therefore, the graph is $|A|$ regular.

Now, we can determine the girth of $\Gamma$.

Theorem 2. Suppose that $H:=C\left(G^{3}\right)$ has index 2. Then the graph $\Gamma$ has girth 3 or 4 . Also the girth is 3 if and only if either

i. $\quad$ There exists $h \in H$ such that $h^{6} \notin Z(G)$ or

ii. $\quad\left\{h^{6} \mid h \in H\right\} \subseteq Z(G)$ and there exist $h, k \in A$ such that $k h^{-1} \in A$, where $A=\left\{h \in H \mid h^{3} \notin Z(G)\right\}$, and $k^{x} \neq h$, for some $x \in G \backslash H$.

Proof. Let $x$ be any element of $G$ outside $H$. First, we note that if $h \in H$, then $x h$ and $h x$ are not adjacent if and only if $h^{6} \in Z(G)$. In fact, if $x h$ and $h x$ are not adjacent, then $(x h h x)^{3}=(h x x h)^{3}$. Since $h x^{2}=x^{2} h$, we have $\left(x h^{2} x\right)^{3}=\left(h x^{2} h\right)^{3}$ implies $\left(x h^{2} x\right)^{3}=\left(h^{2} x^{2}\right)^{3}$ and so $x h^{2} x x h^{2} x x h^{2} x=h^{2} x^{2} h^{2} x^{2} h^{2} x^{2}$. Hence $x h^{6} x^{5}=h^{6} x^{6}$ and $x h^{6}=h^{6} x$. Since for all $u \in H$ we have $u h^{6}=h^{6} u$, it follows that $h^{6} \in Z(G)$. This argument also shows that if $h^{6} \in Z(G)$, then $x h$ and $h x$ are not adjacent.

Suppose that condition (i) holds, that is $h^{6} \in Z(G)$, for some $h \in H$. By Lemma 2, $(x, x h)$ and $(x, h x)$ are edges of $\Gamma$. Since $h^{6} \notin Z(G)$, by observation, $x h$ and $h x$ are adjacent. Thus $(x, x h, h x)$ form a triangle and so $\operatorname{girth}(\Gamma)=3$.

Now suppose that condition (ii) holds, that is $\left\{h^{6} \mid h \in H\right\} \subseteq Z(G)$ and there exist $h, k \in H$ such that $\left\{h^{3}, k^{3},\left(k h^{-1}\right)^{3}\right\} \bigcap Z(G)=\phi$. By Lemma 2, $(x, h x)$ and $(x, k x)$ are edges on $\Gamma$. Also, by Lemma 2, $\left(h x, k h^{-1}(h x)\right)=(h x, k x)$ is an edge of $\Gamma$. Thus, there is a triangle with vertices $x, h x$ and $k x$. Hence, $\operatorname{girth}(\Gamma)=3$

Now suppose that the girth of $\Gamma$ is 3 . If $(x, x h, h x)$ is a triangle, then $h^{6} \notin Z(G)$ and condition (i) holds. So suppose that $\left\{h^{6} \mid h \in H\right\} \subseteq Z(G)$. Given a triangle with vertices $h_{1} x, h_{2} x$ and $h_{3} x$, set $z=h_{1} x$ and setting $k_{2}=h_{2} h_{1}^{-1}$ and $k_{3}=h_{3} h_{1}^{-1}$ the vertices of the triangle become $z, k_{2} z$ and $k_{3} z$. So it might be assumed that any triangle has vertices $x, h x$ and $k x$, where $x \notin H$, $h, k \in H$ and by Lemma $2, h, k \in A$. Viewing the edge $(h x, k x)$ as $\left(h x, k h^{-1}(h x)\right)$, there is also $k h^{-1} \in A$. As, in the case, $(h x, x h)$ is not as edge of $\Gamma$, it must has $k x \neq x h$ so $k^{x} \neq h$. Therefore, the condition (ii) holds.

By Lemma 2, there exists a non-identity element $h \in H$ such that $h^{3} \notin Z(G)$. Suppose that the conditions (i) and (ii) do not hold. Then $h^{6} \in Z(G)$. Since $h^{3} \notin Z(G), \quad$ by Lemma 2, $(x, h x),\left(x, h^{-1 x}\right),(h x, h(h x))=\left(h x, h^{2} x\right)$ are edges of $\Gamma$. Now, if $h^{3}=1$, then since $h^{6} \in Z(G)$ it has been concluded that $h \in Z(G)$, contradicting $h^{3} \notin Z(G)$. Thus, $h^{3} \neq 1$ and, by Lemma $2,\left(h^{-1} x, h^{-1}\left(h^{3} x\right)\right)=\left(h^{-1} x, h^{2} x\right)$ is an edge of $\Gamma$. Hence, $\left\{x, h x, h^{2} x, h^{-1} x\right\}$ form a square. Therefore, $\operatorname{girth}(\Gamma)=4$.

\section{SUMMARY}

In this paper, new graph has been introduced which is the graph related to cubed commutativity degree. It is proven that the graph is complete if and only if its subgroup is abelian of odd order with index two. 


\section{REFERENCES}

Abdollahi, A., Akbari, S. and Maimani, H. R. (2006). Noncommuting graph of a group. J. Algebra, 298, 468-492.

Bertram,E.A., Herzog, M. and Mann, A.(1990).On a graph related to conjugacy classes og groups. Bull. London Math. Soc., 22, 569-575

Bianchi, M., Chillag, D., Mauri, A., Herzog, M. and Scoppola, C. (1992). Application of a graph related to conjugacy classes in finite groups. Arch Math, 58, 126-132.

Bianchi, M., Herzog, M., Pacifici, E. and Saffirio, G. (2012). On the regularity of a graph related to conjugacy classes of groups. European Journal of Combinatorics, 33(7), 14021207 .

Chillag, D., Herzog, M. and Mann, A. (1993). On the diameter of a graph related to conjugacy classes of groups. Bull. London Math. Soc., 25, 255-256.

El-sanfaz, M. A. and Sarmin, N. H. (2015). On the probability that an element of metacyclic 2-group of positive type fixes a set and its generalized conjugacy class graph. Global Journal of Pure Mathematics, 11(2), 899-908.

Erfanian, A. and Tolue, B. (2012). Conjugate graphs of finite groups, Discrete Mathematics, Algorithms and Applications, 4(2)(2), 35-43.

Erfanian, A. and Tolue, B. (2012). Relative non nil- $n$ graphs of finite groups. Science Asia, 38, 201-206.

Moreto, A., Qian, G. and Shi, W. (2005). Finite groups whose conjugacy classes graphs have few vertices. Arch. Math., $85,101-107$.

Omer, S. M. S. and Sarmin, N. H. (2014). The orbit graph for some finite solvable groups. In Proceedings of The $3^{\text {rd }}$ International Conference of Mathematical Sciences, 1602, 863-869. doi:10.1063/1.4882585.

You, X., Qian, G. and Shi, W. (2005). A new graph related to conjugacy classes of finite groups. arXivmath.0510015. 